\title{
Employing the fluorescence anisotropy and quenching kinetics of tryptophan to hunt for residual structures in denatured proteins
}

\author{
SATISH KUMAR and RAJARAM SWAMINATHAN* \\ Department of Biotechnology, Indian Institute of Technology - Guwahati, Guwahati 781039 \\ e-mail: rsw@iitg.ernet.in
}

\begin{abstract}
Residual structures in denatured proteins have acquired importance in recent years owing to their role as protein-folding initiation sites. Locating these structures in proteins has proved quite formidable, requiring techniques like NMR. Here in this report, we take advantage of the ubiquitous presence of tryptophan residues in residual structures to hunt for their presence using steady-state fluorescence spectroscopy. The surface accessibility and rotational dynamics of tryptophan in putative residual structures among ten different proteins, namely glucagon, melittin, subtilisin carlsberg, myelin basic protein, ribonuclease $\mathrm{T}_{1}$, human serum albumin, barstar mutant, bovine serum albumin, lysozyme and Trp-MetAsp-Phe- $\mathrm{NH}_{2}$ peptide, was studied using steady state fluorescence quenching and anisotropy, respectively. Five proteins, namely ribonuclease $\mathrm{T}_{1}$, bovine serum albumin, melittin, barstar and hen egg white lysozyme appear likely to possess tryptophan(s) in hydrophobic clusters based on their reduced bimolecular quenching rates and higher steady-state anisotropy in proportion to their chain length. We also show that the fluorescence emission maximum of tryptophan is insensitive to the presence of residual structures.
\end{abstract}

Keywords. Guanidine hydrochloride; polarization; indole; hydrophobic cluster; iodide; protein folding.

\section{Introduction}

The presence of a non-random structure in proteins, most commonly around a hydrophobic cluster under strongly denaturing conditions, is termed a residual structure. ${ }^{1,2}$ Residual structures are widely believed to act as nucleation sites from where the process of protein folding is likely to originate, thereby reducing the Levinthal search considerably. Considerable evidence for the existence of these structures have come from NMR approaches, beginning from the work of Wuthrich and coworkers. ${ }^{3}$ It has been consistently observed that residual structures in proteins exist around hydrophobic amino acid clusters. Several instances of such clusters involving the Trp residue(s) are known. ${ }^{3-9}$ In one case, the mutation of Trp to Gly is shown to lead to disruption of residual structures. $^{7}$

Locating residual structures in proteins is not easy. Conventional approaches like circular dichroism, in-

\footnotetext{
*For correspondence

Abbreviations: GdnCl, guanidine hydrochloride; NATA, Nacetyl-L-tryptophanamide; RNase $\mathrm{T}_{1}$, ribonuclease $\mathrm{T}_{1}$; $\mathrm{MBP}$, basic myelin; SC, protease subtilisin carlsberg; HSA, human serum albumin; BSA, bovine serum albumin; Trp, tryptophan; W, tryptophan; Gly, glycine
}

frared spectroscopy, fluorescence intensity/lifetime have not been effective in highlighting their presence. Keeping in mind the importance of denatured state to the protein-folding problem, we wish to investigate if alternate approaches, other than NMR, can be employed to locate residual structures. The work reported here is an attempt to address the above question.

The fluorescence from the indole side chain in Trp lends itself to being a convenient spectroscopic probe for the structure and rotational dynamics surrounding the Trp residue in the protein. ${ }^{10,11}$ The bimolecular fluorescence quenching rate constant $\left(k_{q}\right)$ of indole by extrinsic quenchers like iodide reveals a lot about its surface accessibility and how deep it is buried in the protein. ${ }^{12}$ In this regard, iodide is (a) selective in quenching surface exposed Trp residue(s) in a protein, ${ }^{13}$ and (b) unlike acrylamide, ${ }^{14}$ free from static quenching, making it a convenient quencher for steady-state fluorescence studies. The steady-state fluorescence anisotropy $\left(r_{s s}\right)$ of indole can reveal the extent of rotational freedom and dynamics available to the Trp side chain in the excited state. ${ }^{15}$ The $r_{s s}$, however, is dependent on both the fluorescence lifetime and the rotational correlation time of the fluorophore. Changes in $r_{s s}$ can 
be meaningfully correlated with rotational motion only when fluorescence lifetime remains fairly invariant under the same condition. Both the above parameters, namely $k_{q}$ and $r_{s s}$, are sensitive indicators of structure, if any, surrounding the Trp probe.

In this report, we present a systematic study on the accessibility and rotational dynamics of Trp in a series of ten proteins in the presence of $6 \mathrm{M} \mathrm{GdnCl}$, conditions under which residual structures are known to persist. ${ }^{5}$ Using this approach, we demonstrate the ability of fluorescence quenching and anisotropy to pick out residual structures in the vicinity of Trp among denatured proteins.

\section{Materials and methods}

Barstar employed here refers to the W38FW44F mutant which contains a single Trp, W53. The purification of W38FW44F mutant of barstar has been described previously. ${ }^{16}$ NATA, RNase $\mathrm{T}_{1}$ (Aspergillus oryzae), glucagon (mixture of bovine and porcine pancreas), melittin (bee venom), human serum albumin, bovine serum albumin, protease subtilisin carlsberg (bacterial), basic myelin (bovine brain), lysozyme (chicken egg white) and Trp-Met-AspPhe $-\mathrm{NH}_{2} \cdot \mathrm{HCl}$ peptide of highest purity were purchased from Sigma-Aldrich Chemicals Private Limited, New Delhi. All other chemicals employed were of analytical grade.

Steady-state fluorescence intensity was measured using SPEX FluoroMax-3 fluorimeter purchased from Jobin Yvon Inc., USA, having automated Glan Thompson polarizers. For all measurements, excitation was done at $295 \mathrm{~nm}$ ( $1 \mathrm{~nm}$ slitwidth). The fluorescence emission spectrum was collected in the range $310-400 \mathrm{~nm}$ (3 nm slitwidth). For quenching experiments, the integrated fluorescence intensity between 340 and $380 \mathrm{~nm}$ was used to determine $F_{0} / F$ since it gave a flat baseline free from Raman scatter and background fluorescence as determined using blank solutions containing $0.4 \mathrm{M} \mathrm{KI}, 6 \mathrm{M}$ $\mathrm{GdnCl}$ and other components. The medium also contained $0 \cdot 1 \mathrm{mM} \mathrm{Na}_{2} \mathrm{~S}_{2} \mathrm{O}_{3}$ to prevent formation of $I^{3-}$. The fluorescence intensity data are averages of at least three independent measurements. Stern-Volmer constant, $K_{\mathrm{SV}}=k_{q} \tau_{m}$ was determined from the slope of the linear regression fit in figure 1. For all data, the square of Pearson product moment correlation coefficient for the fit was in the range 0.96-0.99, intercept was between 0.92 and 1.05 . Uncertainties in values of $F_{0}$ and $F$ are less than $5 \%$. Mean fluores- cence lifetime, $\tau_{m}$, in the presence of $\sim 6 \mathrm{M} \mathrm{GdnCl}$ was obtained from earlier work for NATA, ${ }^{17}$ Barstar $^{16}$ and others. ${ }^{18}$ The fluorescence lifetime of Trp in BSA and Trp-Met-Asp-Phe- $\mathrm{NH}_{2} \cdot \mathrm{HCl}$ peptide in the presence of $\sim 6 \mathrm{M} \mathrm{GdnCl}$ was determined separately using a technique similar to that described previously. ${ }^{18}$

Steady state fluorescence anisotropy, $r_{s s}$, was experimentally measured for $355 \mathrm{~nm}$ emission (5$10 \mathrm{~nm}$ slitwidth) using the L-format method ${ }^{19}$ incorporating $G$-factor correction. All values reported in table 1 are averages of at least five independent measurements.

To ensure complete denaturation, all proteins were soaked in the denaturant $(\approx 6 \mathrm{M} \mathrm{GdnCl})$ overnight at room temperature prior to the experiment. All protein concentrations were in the range 3-10 $\mu \mathrm{M}$ for quenching experiments and $25-60 \mu \mathrm{M}$ for anisotropy experiments. All samples except melittin were buffered at $\mathrm{pH} 7$ (phosphate). Melittin was buffered at $\mathrm{pH} 8$ (Tris) to avoid tetramer formation. All experiments were performed at $293 \mathrm{~K}$.

\section{Results and discussion}

We have employed the technique of steady state fluorescence quenching and fluorescence anisotropy

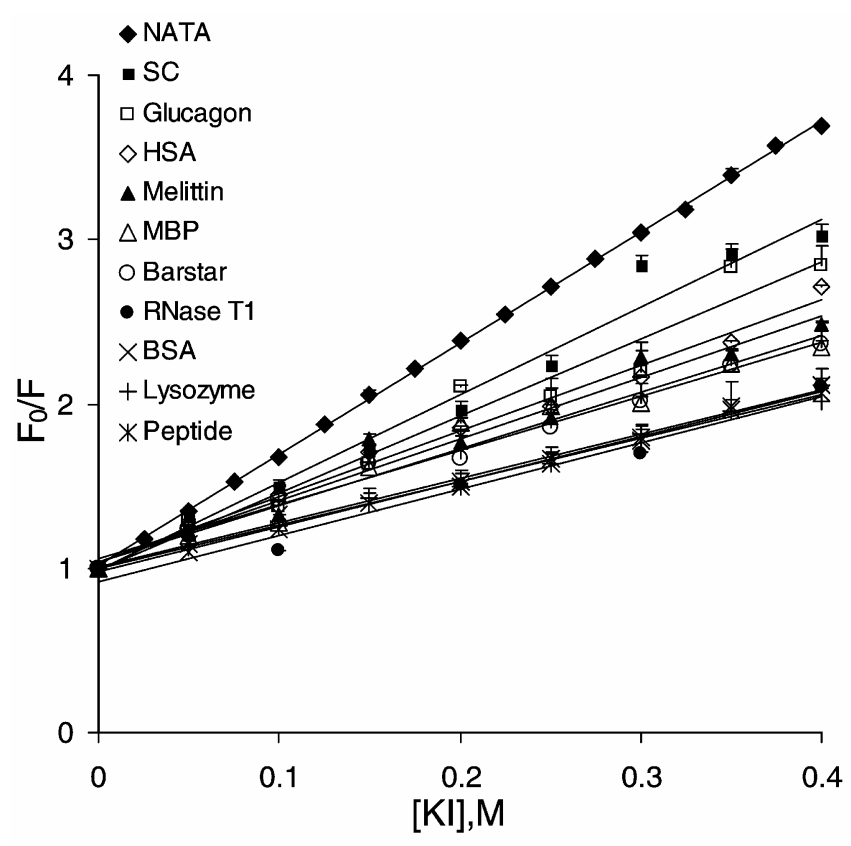

Figure 1. Stern-Volmer plot depicting fluorescence quenching of Trp in NATA and different proteins by iodide in the presence of $6 \mathrm{M} \mathrm{GdnCl}$. See table 1 for SternVolmer constant, $K_{\mathrm{SV}}$. For experimental details see $\S 2$. 
Table 1. Fluorescence quenching, steady state anisotropy and emission spectrum parameters of proteins in $6 \mathrm{M}$ $\mathrm{GdnCl}$. For more details see $\$ 2$.

\begin{tabular}{|c|c|c|c|c|c|c|c|}
\hline Sample & $\begin{array}{c}K_{\mathrm{SV}} \\
\left(\mathrm{M}^{-1}\right)\end{array}$ & $\begin{array}{c}\tau_{m} \\
(\mathrm{~ns})\end{array}$ & $\begin{array}{c}k_{q}^{*}\left(\mathrm{M}^{-1} \mathrm{~s}^{-1}\right) \\
\left(\times 10^{9}\right)\end{array}$ & $r_{s s}$ & $\begin{array}{l}\text { Chain } \\
\text { length }\end{array}$ & $\lambda_{\max } \underset{(\mathrm{nm})}{\text { Emission }}$ & $\begin{array}{c}\text { Position of } \\
W \text { in the chain }\end{array}$ \\
\hline NATA & $6 \cdot 76$ & $2 \cdot 84$ & $2 \cdot 38$ & $0 \cdot 014 \pm 0 \cdot 004$ & 1 & 352 & 1 \\
\hline Melittin & $3 \cdot 73$ & $2 \cdot 43$ & 1.53 & $0.062 \pm 0.002$ & 26 & 352 & 19 \\
\hline Glucagon & $4 \cdot 67$ & $2 \cdot 06$ & $2 \cdot 27$ & $0 \cdot 048 \pm 0.003$ & 29 & 350 & 14 \\
\hline Barstar & $3 \cdot 29$ & $2 \cdot 16$ & 1.52 & $0.095 \pm 0.004$ & 89 & 350 & 53 \\
\hline RNase $T_{1}$ & $2 \cdot 80$ & $2 \cdot 66$ & 1.05 & $0.091 \pm 0.004$ & 104 & 348 & 59 \\
\hline MBP & $3 \cdot 45$ & 1.78 & 1.94 & $0.058 \pm 0.007$ & 169 & 350 & 115 \\
\hline $\mathrm{SC}$ & $5 \cdot 34$ & $2 \cdot 40$ & $2 \cdot 23$ & $0.053 \pm 0.005$ & 274 & 352 & 112 \\
\hline HSA & 3.98 & 1.96 & $2 \cdot 03$ & $0.084 \pm 0.005$ & 585 & 350 & 213 \\
\hline BSA & $2 \cdot 75$ & $2 \cdot 39$ & $1 \cdot 15$ & $0.091 \pm 0.002$ & 583 & 348 & 134,213 \\
\hline Lysozyme & $2 \cdot 63$ & 1.66 & 1.58 & $0 \cdot 102 \pm 0 \cdot 002$ & 129 & 348 & $\begin{array}{c}28,62,63 \\
108,111,123\end{array}$ \\
\hline Trp-Met-Asp-Phe & $2 \cdot 70$ & $0 \cdot 89$ & $3 \cdot 03$ & $0.045 \pm 0.006$ & 4 & 344 & 1 \\
\hline
\end{tabular}

*Values here are to be multiplied by $10^{9}$ for the true value

to investigate the presence of putative residual structures in proteins. Both the parameters, namely Trp accessibility and Trp rotational dynamics are sensitive indicators of any structure that may exist under denaturing conditions. Among the ten proteins employed in our investigation, eight proteins namely, barstar, SC, HSA, melittin, MBP, glucagon, RNase $\mathrm{T}_{1}$ and Trp-Met-Asp-Phe possess only one tryptophan per polypeptide chain, making unambiguous interpretation of the data possible at the molecular level.

Figure 1 shows the Stern-Volmer plot observed for quenching of model compound NATA and eight single Trp proteins by iodide in the presence of $6 \mathrm{M}$ $\mathrm{GdnCl}$. Results are also presented for two multi-tryptophan proteins namely, lysozyme and bovine serum albumin. NATA shows a linear variation of $F_{0} / F$ against iodide concentration, indicating that quenching is purely dynamic in nature, consistent with previous reports. ${ }^{20}$ The presence of static component in quenching would have resulted in an upward curvature owing to the quadratic dependence of $F_{0} / F$ on quencher concentration. The SternVolmer constant, $K_{\mathrm{SV}}$ calculated from the slope of the fitted straight line and bimolecular quenching constant, $k_{q}$, is shown in table 1 . In figure 1 , the $F_{0} / F$ data corresponding to proteins SC and glucagon appear relatively more scattered about their linear regression fit compared to the rest. However, the value of $k_{q}$ for SC and MBP are fairly consistent with values from an earlier report ${ }^{13}\left(2.0 \times 10^{9} \&\right.$ $1.24 \times 10^{9} \mathrm{M}^{-1} \mathrm{~s}^{-1}$ for SC and MBP respectively). A $k_{q}$ value of $1.0 \times 10^{9} \mathrm{M}^{-1} \mathrm{~s}^{-1}$ was observed earlier for wild-type barstar (which contains three tryptophans) too. ${ }^{21}$ Table 1 reveals that all proteins employed in the study possess a lower $k_{q}$ compared to a tiny molecule like NATA and the tetrapeptide Trp-MetAsp-Phe. Interestingly, the hindrance posed by the long flexible swollen polypeptide to the diffusional encounter with iodide, especially when $\mathrm{W}$ is located in the middle of the chain (as in MBP, SC and HSA in table 1) appears negligible. RNase $\mathrm{T}_{1}$ displays a $k_{q}$ that is lower than $50 \%$ of the value observed with NATA. Melittin, barstar, BSA and lysozyme also display fairly low values for the bimolecular quenching constant in comparison to the rest of proteins. Significant amount of shielding from iodide is likely to arise if residual structures exist in the vicinity of $\operatorname{Trp}$ in the above mentioned proteins.

Table 1 shows the steady state fluorescence anisotropy, $\left(r_{s s}\right)$ for the indole ring in eight single Trp proteins, two multi-tryptophan proteins and NATA. A value of 0.014 observed for NATA is consistent with a rotational correlation time $\sim 0.15 \mathrm{~ns}$ (calculated from Perrin equation using $r_{0}=0 \cdot 274^{19}$ ) expected for a tiny molecule like NATA in a mildly viscous medium of $6 \mathrm{M} \mathrm{GdnCl}$. For the tetrapeptide too, a value of 0.045 is consistent with a fast rotational motion, given its short mean fluorescence lifetime $(0.89 \mathrm{~ns})$. It is evident that the mean fluorescence lifetime of Trp in denatured proteins studied here falls within a narrow range between $1.7-2.7 \mathrm{~ns} .{ }^{18}$ Thus we may consider the fluorescence lifetime of Trp to be approximately constant among these proteins and correlate changes in $r_{s s}$ to predominantly changes in Trp rotational dynamics. A striking fea- 
ture in table 1 , is the fairly good correlation between two independent parameters, $k_{q}$ and $r_{s s}$ This is obvious in the case of RNase $T_{1}$, where a significant degree of shielding against iodide quenching and a relatively larger $r_{s s}$ is evident. This implies that the W59 in RNase $T_{1}$ is part of a residual structure. Indeed, evidence for residual structures in this protein has emerged earlier. ${ }^{22}$ Using temperature dependent NOE data, it has been previously shown ${ }^{22}$ that helical region in RNase $T_{1}$ unfolds at a lower temperature compared to $\beta$-sheet $\mathrm{B}$ (where W59 resides). Like many other Trp in hydrophobic clusters, W59 in RNase $T_{1}$ is also buried in a hydrophobic core in the native protein. Among the other single Trp containing proteins, melittin and barstar too possess a relatively ordered Trp as revealed by a $k_{q} \sim 1.5 \times 10^{9} \mathrm{M}^{-1} \mathrm{~s}^{-1}$ and a moderately large $r_{s s}$ in proportion to their chain length. The W53 in barstar is known to be buried in the hydrophobic core. ${ }^{16}$ Previous reports have also indicated the presence of residual structure around W53 in barstar. ${ }^{23-25}$ Lysozyme, which has six tryptophans, has a low $k_{q}$ and a longer correlation time. It has been shown using $\mathrm{NMR}^{7}$ that, four of the six Trps in lysozyme are indeed, part of hydrophobic clusters in $8 \mathrm{M}$ urea.

Melittin which is almost similar in size to peptide glucagon, shows a significantly higher steady state anisotropy and lower $k_{q}$ in contrast to glucagon. This strengthens the possibility that Trp in melittin is part of a residual structure.

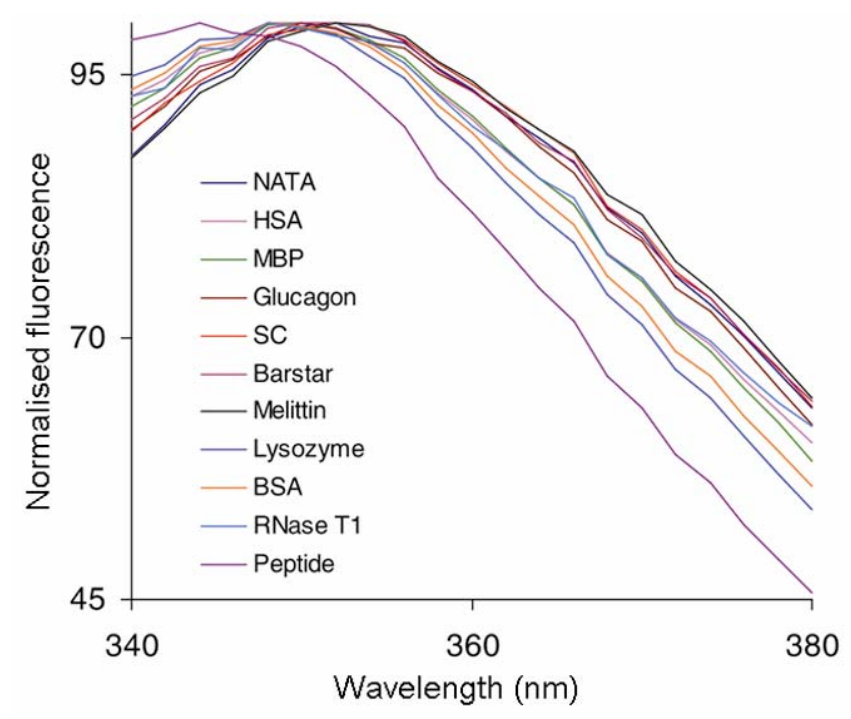

Figure 2. Corrected fluorescence emission spectra of Trp in different proteins in the presence of $6 \mathrm{M} \mathrm{GdnCl}$. See table 1 for wavelength corresponding to emission maximum. For experimental details see $\S 2$.
It is interesting to compare the data in table 1 for BSA and HSA which are nearly similar in polypeptide chain length. While the sole Trp in HSA has a $k_{q} \sim 2.0 \times 10^{9}$, the overall $k_{q}$ of two tryptophans in BSA is relatively less $\left(\approx 1 \cdot 1 \times 10^{9}\right)$, although in both cases the position of tryptophan residues in the polypeptide are well in the interior. The $r_{s s}$ observed for BSA is marginally higher compared to HSA, but importantly the mean fluorescence lifetime for BSA is also higher suggesting a slower rotational correlation time compared to HSA. Based on these observations it is likely that at least one of the tryptophan residues in BSA is part of a residual structure.

The wavelength corresponding to fluorescence emission maximum for indole side chain in denatured proteins forms yet another parameter to estimate the exposure of the indole ring to the solvent. With the exception of the peptide which shows a peak near $344 \mathrm{~nm}$, almost all other proteins in our study revealed emission maxima between 348 and $352 \mathrm{~nm}$ in the denatured state (figure 2 and table 1), which is close to that observed for NATA, indicating that indole is solvent exposed in all the proteins studied. We know from quenching data that the indole in RNase $\mathrm{T}_{1}, \mathrm{BSA}$, melittin, barstar and lysozyme is not freely accessible to a large anion like iodide. The absence of correlation between indole emission maxima and rate of iodide quenching is clearly evident in the case of the tetrapeptide, which has the lowest emission maximum and highest bimolecular quenching constant. Clearly, iodide quenching experiments provide a superior alternative to locate residual structures compared to fluorescence emission maxima.

Fluorescence quenching by acrylamide ${ }^{14}$ but not iodide, has been used earlier for probing residual structures. Our data show that in contrast to acrylamide, iodide permits easy analysis of quenching data, owing to the absence of static quenching.

\section{Conclusion}

Our results show that fluorescence bimolecular quenching-rate constant and steady-state anisotropy of Trp, can serve as useful parameters to search and locate residual structures in the vicinity of Trp in denatured proteins. Unlike the fluorescence emission maxima, these parameters are sensitive indicators of the structural order surrounding Trp in denatured proteins. Further experiments employing time-resolved fluorescence anisotropy are required 
to obtain more specific details on the rotational motion especially for the proteins identified by us to possess residual structure in the vicinity of tryptophan.

\section{Acknowledgements}

RS wishes to thank Prof J B Udgaonkar, National Centre of Biological Sciences, Bangalore for W38FW44F barstar mutant. Financial support from the Ministry of Human Resource Development, New Delhi is gratefully acknowledged. We wish to thank Prof G Krishnamoorthy, Tata Institute of Fundamental Research, Mumbai for the fluorescence lifetime measurements of tryptophan in BSA and tetrapeptide.

\section{References}

1. Hammarstrom P and Carlsson U 2000 Biochem. Biophys. Res. Commun. 276393

2. Dill K A and Shortle D 1991 Annu. Rev. Biochem. 60 795

3. Neri D, Billeter M, Wider G and Wuthrich K 1992 Science 2571559

4. Tafer H, Hiller S, Hilty C, Fernandez $\mathrm{C}$ and Wuthrich K 2004 Biochemistry 43860

5. Pearce M C, Cabrita L D, Rubin H, Gore M G and Bottomley S P 2004 Biochem. Biophys. Res. Commun. 324729

6. Crowhurst K A, Tollinger M and Forman-Kay J D 2002 J. Mol. Biol. 322163

7. Klein-Seetharaman J, Oikawa M, Grimshaw S B, Wirmer J, Duchardt E, Ueda T, Imoto T, Smith L J, Dobson C M and Schwalbe H 2002 Science 2951719

8. Evans P A, Topping K D, Woolfson D N and Dobson C M 1991 Proteins: Struct. Funct. Genet. 9248
9. Ropson I J and Freiden C 1992 Proc. Natl. Acad. Sci. USA 897222

10. Lakowicz J R (ed.) 2000 Topics in fluorescence spectroscopy (London: Kluwer Academic/Plenum) vol. 6

11. Beechem J M and Brand L 1985 Annu. Rev. Biochem. 5443

12. Eftink M R 1991 Fluorescence quenching: Theory and applications. In Topics in fluorescence spectroscopy (ed.) J R Lakowicz (New York: Plenum) vol. 2, pp 53-126

13. Lakshmikanth G S and Krishnamoorthy G 1999 Biophys. J. 771100

14. Garcia P, Merola F, Receveur V, Blandin P, Minard P and Desmadril M 1998 Biochemistry 377444

15. Steiner R F 1991 Fluorescence anisotropy: Theory and application. In Topics of fluorescence spectroscopy (ed.) J R Lakowicz (New York: Plenum) vol. 2, pp $1-52$

16. Swaminathan R, Nath U, Udgaonkar J B, Periasamy $\mathrm{N}$ and Krishnamoorthy G 1996 Biochemistry 359150

17. Swaminathan R, Periasamy N, Udgaonkar J B and Krishnamoorthy G 1994 J. Phys. Chem. 989270

18. Swaminathan R, Krishnamoorthy G and Periasamy N 1994 Biophys. J. 672013

19. Lakowicz J R 1996 Principles of fluorescence spectroscopy (New York: Plenum) pp. 297-298

20. Maity H and Jarori G K 2002 Physiol. Chem. Phys. \& Med. NMR 3443

21. Swaminathan R 1995 Time-resolved fluorescence studies in biological macromolecules, $\mathrm{Ph} \mathrm{D}$ thesis, University of Bombay, Mumbai

22. Matsuura H, Shimotakahara S, Sakuma C, Tashiro M, Shindo H, Mochizuki K, Yamagishi A, Kojima M and Takahashi K 2004 Biol. Chem. 3851157

23. Bhavesh N S, Juneja J, Udgaonkar J B and Hosur R V 2004 Protein Sci. 133085

24. Wong K B, Freund S M V and Fersht A R $1996 \mathrm{~J}$. Mol. Biol. 259805

25. Saxena A M, Udgaonkar J B and Krishnamoorthy G 2006 J. Mol. Biol. 359174 